\title{
Nocardia infection in AIDS: a clinical and microbiological challenge
}

\author{
R J Coker, G Bignardi, P Horner, M Savage, T Cook, D Tomlinson, J Weber
}

\begin{abstract}
A case of Nocardia asteroides pneumonia was diagnosed after death in a patient with AIDS. Six sputum cultures and one bronchoalveolar lavage fluid contained no pathogens, and no growth was obtained from one pleural fluid aspirate. None of these specimens was incubated for more than two days. Extended incubation for mycobacteria also failed to help in the diagnosis. $\boldsymbol{N}$ asteroides was isolated from pus taken from the lung cavity during the post mortem examination.

It is suggested that if nocardiosis enters the differential diagnosis all specimens should be cultured for at least two weeks and the use of selective media be considered. This case highlights the need for clinicians to maintain a high index of suspicion for this pathogen.
\end{abstract}

Nocardia asteroides infection affects mainly debilitated or immunocompromised patients. However, $N$ asteroides seems to be a rare pathogen in patients with AIDS. There have been 14 cases reported in English. ${ }^{1}$ The treatment of choice is generally considered to be prolonged administration of a sulphonamide, though which one varies. Unfortunately adverse reactions to sulphonamides, particularly co-trimoxazole, occur in about $65 \%$ of such patients taking them for Pneumocystis carinii pneumonia (PCP), and in $20-25 \%$ of such patients these drugs have to be discontinued. $^{2}$

The clinical and radiological findings of $N$ asteroides infection are non-specific. However, isolation of this slow grower from specimens submitted for culture is difficult unless the diagnosis is suspected. First, the incubation time needs to be extended (Nocardia spp may not be isolated from routine plates which are incubated for only one to two days) but, even if this is done, Nocardia spp can be outgrown and masked by the normal flora present in sputum and even in bronchoalveolar lavage specimens.

None of the selective media normally available in most microbiology laboratories is suitable for the isolation of Nocardia spp. Neisseria gonorrhoeae media usually contain trimethoprim and would be suitable only if this was omitted. ${ }^{3}$ Saboraud media are unsuitable because they usually contain chloramphenicol. ${ }^{4}$ Growth on mycobacterial media may occur but be adversely affected by the preliminary decontamination procedures. ${ }^{5}$

In this report we describe a patient with AIDS and $N$ asteroides pneumonia in whom routine cultures failed to isolate the organism. The diagnosis was only made posthumously, and as a result of this case we reviewed our culture techniques.

\section{Case report}

A 24 year old homosexual man was first diagnosed as being infected with the human immunodeficiency virus (HIV) in June 1989. He began treatment for tuberculosis with rifampicin, isoniazid, pyrazinamide and pyridoxine after a right upper lobe cavitating lesion was noted on a chest $x$ ray picture. Auramine stains and cultures of bronchoscopy specimens for Mycobacterium tuberculosis were negative. AIDS was diagnosed in September 1990 when he developed cytomegalovirus (CMV) retinitis. He had an appendicectomy in December 1990 which was unrelated to any opportunistic infection. He was admitted in March 1991 with colicky abdominal pain, right sided pleuritic chest pain, and a cough productive of green sputum. On admission he was receiving zidovudine $200 \mathrm{mg}$ daily, fluconazole $50 \mathrm{mg}$ daily, rifampicin $480 \mathrm{mg}$ daily, isoniazid 200 $\mathrm{mg}$ daily, and pyridoxine $10 \mathrm{mg}$ daily. He was also taking dapsone $100 \mathrm{mg}$ and pyrimethamine $25 \mathrm{mg}$ both twice weekly as prophylaxis for PCP.

Examination showed that he was dehydrated, cachexic, and feverish $\left(38^{\circ} \mathrm{C}\right)$. He had right sided abdominal tenderness, guarding, and bowel sounds suggestive of obstruction. His CMV retinitis was inactive. A chest radiograph revealed right mid-zone consolidation, though no evidence of the cavitation noted before, and an abdominal radiograph showed multiple distended thin walled small bowel loops.

A laparotomy was performed and adhesions causing the obstruction were divided. His gastrointestinal symptoms resolved, but his pleuritic chest pain persisted associated with signs of right mid-zone collapse, consolidation, and an effusion, which were confirmed radiographically. No pathogens were isolated from six sputum cultures and one bronchoalveolar lavage fluid and no growth at all was obtained from one pleural fluid aspirate. All specimens were cultured on the following media: McConkey agar incubated at $37^{\circ} \mathrm{C}$ aerobically for one 
day; blood agar and chocolate agar incubated at $37^{\circ} \mathrm{C}$ in a carbon dioxide incubator for two days; Saboraud agar with chloramphenicol incubated at $30^{\circ} \mathrm{C}$ aerobically for five days; and blood agar incubated at $37^{\circ} \mathrm{C}$ anaerobically for two days (the last was used only for the pleural fluid). None of these specimens was incubated for more than two days according to our routine practice. Seven blood cultures, processed with a non-radiometric Bactec system, were all negative. Six sputum specimens, one bronchoalveolar lavage fluid, and one pleural fluid aspirate were negative after culture for mycobacteria (using Lowenstein-Jensen slopes with and without pyruvic acid, liquid Kirchner's medium with selective antibiotics, and the Bactec radiometric TB system after addition of the antimicrobial supplement to the Bactec 12B medium). However, the pleural fluid grew non-acid fast bacilli after four weeks (both in Kirchner's and in the Bactec 12B bottles). These were thought to be contaminants. Despite empirical antibiotic treatment with co-trimoxazole, ceftazidime and gentamicin, ciprofloxacin, and subsequently piperacillin, gentamicin, and metronidazole, he continued to deteriorate. He died 11 weeks after the laparotomy.

$N$ asteroides was isolated from pus taken from the lung cavity during the post mortem examination, after incubation of blood agar and chocolate agar in the carbon dioxide incubator for two days. After his death the Kirchner's culture of the pleural fluid was re-examined at the end of the twelfth week of incubation for mycobacteria: the non-acid fast bacteria which had been assumed to be contaminants after four weeks of incubation showed branching on a Gram film and were subsequently identified as $N$ asteroides. The isolate was identified as $N$ asteroides by colonial and microscopic appearance, acid fastness with modified decolorisation as suggested by Kinyoun, and biochemical tests (casein digestion, urea hydrolysis, tyrosine xanthine and hypoxanthine decomposition). Post mortem examination showed a 4 $\mathrm{cm}$ diameter abscess in the lower lobe and a $1.5 \mathrm{~cm}$ diameter abscess in the upper lobe of the right lung. There were extensive smaller abscesses throughout the right lung. Histological analysis showed that the abscesses contained abundant colonies of a slender Gram positive, branched filamentous organism with the typical appearance of Nocardia. Colonies were also present within bronchioles.

\section{Discussion}

Nocardia infection associated with HIV is rare, though why this should be is unknown. ${ }^{6}$ Nocardia infection may be more common than suspected, and perhaps we either fail to detect it because of flaws in our culture techniques or because we treat it with antibiotics directed at other pathogens. Both treatment and prophylaxis of PCP with co-trimoxazole is common, and sulphonomides are the drugs of choice in Nocardia sp infection. The frequent use of cotrimoxazole may partly explain the low incidence of this infection. The use of dapsone for PCP prophylaxis may also reduce the incidence; nocardiosis has responded to treatment with this drug. ${ }^{7}$

The clinical and radiological findings of $N$ asteroides infection are not specific. However, isolation requires specific and more cumbersome culture techniques which cannot be applied to all specimens. We suggest that in patients with or without AIDS nocardiosis should be considered whenever pleuropulmonary infection occurs in the absence of detectable pathogens from multiple respiratory specimens, particularly if the patient fails to respond to antibiotic regimens which are not effective against $N$ asteroides. If nocardiosis enters the differential diagnosis all specimens should be cultured for at least two weeks and the use of selective media might be considered.

Because of its low prevalence nocardiosis is not likely to be suspected until after negative cultures of multiple specimens. Specimens such as bronchoalveolar lavage fluid cannot easily be repeated. As an interim measure we have extended the incubation time of all bronchoalveolar lavage and pleural fluid specimens to two weeks. We intend to review the cost effectiveness of this measure after a period of time.

The accidental isolation of $N$ asteroides from cultures for mycobacteria has been reported by others $^{1}$ as well. The possibility of isolating Nocardia spp from mycobacterial cultures should not be overlooked, although these cultures should not be relied on as the only method as Nocardia spp may not survive the initial decontamination. ${ }^{5} N$ asteroides is not acid fast when the strong decolorisation of the routine Ziehl-Neelsen stain is used, therefore, we suggest that non-acid fast isolates should be examined carefully for the presence of branching before being discarded as contaminants.

1 Kim J, Minamoto GY, Grieco MH. Nocardial infection as a complication of AIDS: report of six cases and review. Rev Infect Dis 1991;13:624-9.

2 Kovacs JA, Hiemenz JW, Macher AM, et al. Pneumocystis carinii pneumonia: A comparison between patients with the acquired immunodeficiency syndrome and patients the acquired immunodeficiency syndrome and patients 1984;100:663-71.

3 Murray PR, Niles AC, Heeren RL. Modified Thayer-Martin medium for recovery of Nocardia species from contamimedium for recovery of Nocardia species from conta

4 Bach MC, Sabath LD, Finland M. Susceptibility of Nocardia asteroides to 45 antimicrobial agents in vitro. Antimicrob Agents Chemother 1973;3:1-8.

5 Murray PR, Heeren RL, Niles AC. Effect of decontamination procedures on recovery of Nocardia spp. $\mathcal{f}$ Clin Microbiol 1987;25:2010-1.

6 Lynn WE, Whyte M, Weber J. Nocardia, mycobacteria and AIDS. AIDS 1989;3:766-7.

7 Rogers R, Muller S. Treatment of actinomycetoma with dapsone. Arch Dermatol 1974;109:529-34. 